\title{
Immunotherapy in first line for extensive-stage small-cell lung cancer: another piece is going to fill the puzzle?
}

\author{
Francesco Passiglia, Silvia Novello \\ Department of Oncology, University of Turin, San Luigi Hospital, Orbassano, Italy \\ Correspondence to: Silvia Novello, MD, PhD, Department of Oncology, University of Turin, San Luigi Hospital, Orbassano, Italy. \\ Email: silvia.novello@unito.it. \\ Provenance: This is an invited Editorial commissioned by Section Editor Song Xu, MD, PhD (Department of Lung Cancer Surgery, Tianjin Medical \\ University General Hospital, Tianjin, China; Tianjin Key Laboratory of Lung Cancer Metastasis and Tumor Microenvironment, Lung Cancer \\ Institute, Tianjin, China). \\ Comment on: Horn L, Mansfield AS, Szczęsna A, et al. First-Line Atezolizumab plus Chemotherapy in Extensive-Stage Small-Cell Lung Cancer. N \\ Engl J Med 2018;379:2220-9.
}

Submitted Nov 30, 2018. Accepted for publication Dec 10, 2018.

doi: $10.21037 /$ atm.2018.12.25

View this article at: http://dx.doi.org/10.21037/atm.2018.12.25

In The New England fournal of Medicine, Horn and colleagues have recently published the results of the IMPower133, a randomized, placebo-controlled, phase III trial, combining the programmed-death ligand 1 (PD-L1) inhibitor atezolizumab or placebo with carboplatin and etoposide in treatment-naive patients with extensivestage small-cell lung cancer (SCLC) (1). The study met its co-primary end-points, showing a significant increase of progression free survival (PFS) in favour of atezolizumab as compared to placebo arm [5.2 vs. 4.3 months; hazard ratio (HR): $0.77 ; 95 \% \mathrm{CI}, 0.62$ to $0.96 ; \mathrm{P}=0.02$ ]. The addition of atezolizumab to chemotherapy resulted also in a significantly longer overall survival (OS) in the intentionto-treat population (12.3 vs. 10.3 months; HR: 0.70; 95\% $\mathrm{CI}, 0.54$ to $0.91 ; \mathrm{P}=0.007)$, while no differences in response rate $(60.2 \%$ vs. $64.4 \%)$ as well as in the duration of response (4.2 vs. 3.9 months) were observed between the two treatment arms. The survival benefit was confirmed across all pre-defined subgroups selected by clinical, pathological and molecular characteristics. As attended, the percentage of patients who reported immune-related adverse-events (AEs) was significantly higher $(39.9 \%$ vs. $24.5 \%)$ in the atezolizumab arm, with grade 1-2 rash and hypothyroidism being the most common. Finally, the percentage of AEs leading to withdrawal from any treatment nearly tripled with atezolizumab as compared to placebo arm.

Since SCLC is almost always smoking-induced and genetically instable, it has been historically considered a potential good candidate for immunotherapy (2). The notion that paraneoplastic syndromes are often accompanied by T-cell mediated antitumor immunity (3), together with the recent evidence that SCLC has one of the highest rate of mutation burden (4), have further enhanced the hope for an efficacious application of checkpoint inhibitors in SCLC treatment. Both PD-1 inhibitors nivolumab $(5,6)$ and pembrolizumab (7) as well as the antiPD-L1 atezolizumab (8) have shown very promising activity and tolerable safety profile in phase I studies including heavily pre-treated patients with extensive-stage SCLC. Recently, the KEYNOTE-158 study (9) confirmed single agent pembrolizumab as an effective treatment option in about 100 patients with relapsed SCLC, showing an objective response rate (ORR) of $18.7 \%$, median PFS of 2.0 months, and median OS of 9.1 months. Conversely the phase 3 randomized CheckMate-331 study did not meet primary end-point of OS with nivolumab versus single agent chemotherapy in previously treated SCLC patients. The majority of phase II-III trials moving checkpoint inhibitors in front-line setting failed to demonstrate any relevant impact on patients' survival. Particularly, a phase II single arm study demonstrated that maintenance therapy with pembrolizumab after frontline platinumetoposide did not improve survival outcomes of 45 patients with extensive stage SCLC (10) as compared to historical 
control. Similarly the CheckMate 451 study did not show any survival benefit with nivolumab plus ipilimumab versus placebo as maintenance therapy in patients with extensive-stage SCLC who completed first-line platinumchemotherapy. Finally, the addition of the CTLA-4 inhibitor ipilimumab to first-line platinum etoposide showed no improved efficacy as compared to chemotherapy alone (11). The results of the IMPower133 trial interrupted the negative trend of upfront immunotherapy in SCLC. As recently observed in non-small cell lung cancer (NSCLC) $(12,13)$, such trial demonstrated that combining checkpoint inhibitors with platinum chemotherapy is an effective and tolerable strategy also in this "recalcitrant" disease. Since the advent of platinum-chemotherapy in 1990s, this is the first study showing a modest, but significant improvement in frontline SCLC standard of care. Although the median OS improvement produced by the addition of atezolizumab is just equal to 2 months, the HR of 0.70 along with an OS increase higher than $10 \%$ at 1 year in the atezolizumab group, resulted in a preliminary score of 2 according to the last version of the European Society of Medical Oncology (ESMO) Magnitude of Clinical Benefit Scale (14), which could be further upgraded if a significant OS increase at 2 years will be confirmed. For this reason, final OS data as well as long term follow-up will be crucial to determine the proportion of patients who will experience long-term benefit from combination therapy, and to definitively establish the clinical impact derived from the addition of atezolizumab to first-line platinum chemotherapy. The main critics regard the design of the trial. Although the control arm, including 4 cycles of carboplatin plus etoposide, performed as predicted, however a maximum of 6 cycles of platinumchemotherapy is currently recommended by all international guidelines $(15,16)$ and this is the common approach in real-world practice. Furthermore, the specific impact of immune-checkpoint inhibitor maintenance therapy on the final OS observed in the IMPower133 remained unclear. Finally, only $7.4 \%$ of patients in the control arm received atezolizumab in second-line, thus we currently do not know whether upfront combination is more effective than sequential treatment in the overall SCLC population. This study clearly showed, once again, as about $50 \%$ of patients with extensive-stage SCLC will never receive second-line therapy after platinum-combinations because of a rapid worsening of clinical conditions, suggesting that the upfront use of atezolizumab could benefit a larger population than if it is used in later lines of treatment.
Concerning the effectiveness of immuno-chemotherapy combination in SCLC patients with brain metastasis, although the subgroup analysis of the IMPower133 showed that they do not derive any benefit from the addition of atezolizumab, the low number of patients included do not allow to draw any definitive conclusions, ensuring further investigation in dedicated trials. The biological mechanisms underlying the effectiveness of atezolizumabchemotherapy regimen as well as the ipilimumab CTLA-4 inhibition failure in a similar SCLC naive population remain a hot topic for translational research. Both CTLA-4 and PD-1 are inhibitory checkpoints playing a key role in the modulation of anti-tumor immune response. In detail, CTLA-4 is implicated in the activation stage of T-cell priming, occurring in regional secondary lymphoid organs, whereas PD-1 acts in the secondary step of T-cell activation process, binding its natural ligands, PD-L1 and PD-L2, which are expressed on tumor, stroma, and immune cells located in the tumor microenvironment $(17,18)$. The understanding of such biological mechanisms may provide a partial explanation to the survival differences observed in the randomized clinical trials, ensuring further investigation in translational studies.

Developing biomarker-driven treatment strategies is an actual challenge of clinical research, involving also trials with immunotherapy. The significant correlation between tumor mutational burden (TMB) and immunotherapy activity observed in pre-treated patients with SCLC (19), was not confirmed in the IMPower133 trial, since the analysis of blood-based TMB levels was not predictive of atezolizumab benefit both in terms of PFS and OS (1), ultimately questioning the utility of TMB in the selection of SCLC patient candidates to upfront combination. The predictive role of tumor PD-L1 expression has not been evaluated in the IMPower133 trial, since data emerging from phase I studies $(5,7)$ clearly demonstrated that PD-L1 has generally low expression on tumor cells and did not correlate to immunotherapy efficacy. Conversely, the expression of PD-L1 in the stroma seems to be higher and significantly associated with survival outcomes of pre-treated patients included in the KEYNOTE-028 and 158 trials $(7,8)$, and its predictive value is currently investigated in the first-line KEYNOTE-604 study comparing Pembrolizumab plus platinum-chemotherapy $v s$. chemotherapy alone in extensive-stage, naive SCLC patients. Even if crucial, the biomarker-driven treatment strategy will inevitably meet several criticisms, considering the limited, low quality, and 
often cytological samples that are almost always used to obtain SCLC diagnosis, likely imposing a radical change of tissue collection practice. In conclusion, the study of Horn et al. represents a significant attempt to the improvement of upfront SCLC therapy. Adding atezolizumab to platinumchemotherapy produced a statistically significant and durable increase of OS together with a modest increment of immune-related toxicities, emerging as new potential standard of care in the treatment of this high aggressive and poor prognosis disease. However, to clearly establish also a "clinical significant impact", final OS data as well as longterm follow-up will be crucial. Additional data supporting the use of upfront immune-chemotherapy combinations are eagerly awaited and the ongoing randomized phase III KEYNOTE-604 and CASPIAN studies, respectively evaluating the addition of pembrolizumab or durvalumab/ tremelimumab to upfront platinum-combinations will provide important results within next year. Considering the advent of new promising drugs/combinations, the main challenge will be how to integrate all these agents and ultimately fill the puzzle of SCLC treatment.

\section{Acknowledgements}

None.

\section{Footnote}

Conflicts of Interest: The authors have no conflicts of interest to declare.

\section{References}

1. Horn L, Mansfield AS, Szczęsna A, et al. First-Line Atezolizumab plus Chemotherapy in Extensive-Stage Small-Cell Lung Cancer. N Engl J Med 2018;379:2220-9.

2. Bunn PA, Minna JD, Augustyn A, et al. Small Cell Lung Cancer: Can Recent Advances in Biology and Molecular Biology Be Translated into Improved Outcomes? J Thorac Oncol 2016;11:453-74.

3. Roberts WK, Deluca IJ, Thomas A, et al. Patients with lung cancer and paraneoplastic Hu syndrome harbor HuD-specific type $2 \mathrm{CD} 8+\mathrm{T}$ cells. J Clin Invest 2009;119:2042-51.

4. Peifer M, Fernández-Cuesta L, Sos ML, et al. Integrative genome analyses identify key somatic driver mutations of small-cell lung cancer. Nat Genet 2012;44:1104-10.
5. Antonia SJ, López-Martin JA, Bendell J, et al. Nivolumab alone and nivolumab plus ipilimumab in recurrent smallcell lung cancer (CheckMate 032): a multicentre, openlabel, phase 1/2 trial. Lancet Oncol 2016;17:883-95.

6. Ready N, Farago AF, de Braud F, et al. Third-Line Nivolumab Monotherapy in Recurrent SCLC: CheckMate 032. J Thorac Oncol 2018. [Epub ahead of print].

7. Ott PA, Elez E, Hiret S, et al. Pembrolizumab in Patients With Extensive-Stage Small-Cell Lung Cancer: Results From the Phase Ib KEYNOTE-028 Study. J Clin Oncol 2017;35:3823-9.

8. Sequist LV CA, Gilbert J, et al. Clinical activity, safety and predictive biomarkers results from a phase Ia atezolizumab (atezo) trial in extensive-stage small cell lung cancer (ESSCLC). Ann Oncol 2016;27:Suppl 6:1425PD.

9. Chung HC, Lopez-Martin JA, Kao SC. et al. Phase 2 study of pembrolizumab in advanced small-cell lung cancer (SCLC): KEYNOTE-158. J Clin Oncol 2018;36:8506.

10. Gadgeel SM, Pennell NA, Fidler MJ, et al. Phase II Study of Maintenance Pembrolizumab in Patients with Extensive-Stage Small Cell Lung Cancer (SCLC). J Thorac Oncol 2018;13:1393-9.

11. Reck M, Bondarenko I, Luft A, et al. Ipilimumab in combination with paclitaxel and carboplatin as first-line therapy in extensive-disease-small-cell lung cancer: results from a randomized, double-blind, multicenter phase 2 trial. Ann Oncol 2013;24:75-83.

12. Gandhi L, Rodríguez-Abreu D, Gadgeel S, et al. Pembrolizumab plus Chemotherapy in Metastatic Non-Small-Cell Lung Cancer. N Engl J Med 2018;378:2078-92.

13. Paz-Ares L, Luft A, Vicente D, et al. Pembrolizumab plus Chemotherapy for Squamous Non-Small-Cell Lung Cancer. N Engl J Med 2018;379:2040-51.

14. Cherny NI, Dafni U, Bogaerts J, et al. ESMO-Magnitude of Clinical Benefit Scale version 1.1. Ann Oncol 2017;28:2340-66.

15. Kalemkerian GP, Loo BW, Akerley W, et al. NCCN Guidelines Insights: Small Cell Lung Cancer, Version 2.2018. J Natl Compr Canc Netw 2018;16:1171-82.

16. Planchard D, Popat S, Kerr K, et al. Metastatic non-small cell lung cancer: ESMO Clinical Practice Guidelines for diagnosis, treatment and follow-up. Ann Oncol 2018;29:iv192-237.

17. Pardoll DM. The blockade of immune checkpoints in cancer immunotherapy. Nat Rev Cancer 2012;12:252-64.

18. Wherry EJ, Kurachi M. Molecular and cellular insights 
into T cell exhaustion. Nat Rev Immunol 2015;15:486-99.

19. Hellmann MD, Callahan MK, Awad MM, et al.

Tumor Mutational Burden and Efficacy of Nivolumab

Cite this article as: Passiglia F, Novello S. Immunotherapy in first line for extensive-stage small-cell lung cancer: another piece is going to fill the puzzle? Ann Transl Med 2018;6(Suppl 2):S120. doi: $10.21037 / \mathrm{atm} .2018 .12 .25$
Monotherapy and in Combination with Ipilimumab in Small-Cell Lung Cancer. Cancer Cell 2018;33:853-61.e4. 